\title{
Rationalität und Solidarität - intensive Existenzen in möglichen Sehnsuchtsräumen
}

Yvonne Hütter-Almerigi

\section{OpenEdition}

1 Journals

Electronic version

URL: http://journals.openedition.org/ejpap/2167

DOI: 10.4000/ejpap.2167

ISSN: 2036-4091

Publisher

Associazione Pragma

Electronic reference

Yvonne Hütter-Almerigi, « Rationalität und Solidarität - intensive Existenzen in möglichen

Sehnsuchtsräumen », European Journal of Pragmatism and American Philosophy [Online], XII-2 | 2020,

Online since 14 December 2020, connection on 15 December 2020. URL : http://

journals.openedition.org/ejpap/2167 ; DOI : https://doi.org/10.4000/ejpap.2167

This text was automatically generated on 15 décembre 2020 .

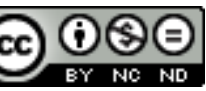

Author retains copyright and grants the European Journal of Pragmatism and American Philosophy right of first publication with the work simultaneously licensed under a Creative Commons AttributionNonCommercial-NoDerivatives 4.0 International License. 


\title{
Rationalität und Solidarität - intensive Existenzen in möglichen Sehnsuchtsräumen
}

\author{
Yvonne Hütter-Almerigi
}

\section{AUTHOR'S NOTE}

This article is part of a project that has received funding from the European Union's Horizon 2020 research and innovation programme under the Marie Skłodowska Curie grant agreement $\mathrm{N}^{\circ} 832636$.

1 Michael Hampe hat ein dringliches und wichtiges Buch geschrieben, in dem er sich, unter anderem, auf Rortys Thesen zur Grausamkeit bezieht. Ich lese und kommentiere Hampes Buch im Folgenden vornehmlich aus Sicht der Rorty-Expertin. Hampe hat ein präzises und unaufgeregtes Verständnis von Rortys Werk. Meine Lesart wird daher nicht Hampes Thesen zuwiderlaufen. Es handelt sich eher um Komplementierung und um Fokusverschiebung rund um die zentrale These, dass Hampes Vorschlag eine Schlagseite hat.

Diese Schlagseite betrifft Hampes Antworten auf zwei miteinander verbundene Fragenkomplexe: 1. Wie grausam ist und darf die (wehrhafte) Aufklärung sein? Worin genau besteht zudem das Grausamkeitspotential? 2. Was minimiert Grausamkeit? Hampes Antworten auf beide Fragenkomplexe scheinen eine Art Askese-Tendenz zu beinhalten - pro Ratio und gegen die Affekte ${ }^{1}$-, die in bester kantischer Tradition steht und deren Komplementierung ich mit Rorty anregen möchte.

3 Zunächst scheint indes, vor der Argumentation, kurz eine Abklärung der Grundintuitionen am Platz, die folgend auch die Perspektive der Argumentation färben werden. Ich teile, wie wahrscheinlich viele, Michael Hampes Gefühl der Dringlichkeit. Krieg in Europa scheint wieder möglich $(7,42)$. Ich teile nicht Michael Hampes 
Diagnose, dass der Hauptgrund für diese Möglichkeit ist, dass die Menschen nicht wissen, dass sie ihr Schicksal in der Hand haben und sich dem Fatalismus hingeben. Ich glaube, das Problem ist eher, dass die sehr wenigen Menschen mit Macht (im Sinne von Entscheidungsgewalt) genau wissen, dass sie die Geschichte mehr oder weniger nach ihrem Gutdünken schreiben können und diese Möglichkeit auch exzessiv nutzen, während es die anderen, ohne Macht, tatsächlich nicht können, und ihnen zu erzählen, dass sie es könnten, etwas Perfides hat, weil die Machtlosen dann nämlich nicht nur machtlos sind, sondern daran auch noch selbst schuld. Diese Art meiner von links kommenden Kritik ist natürlich genauso vorhersehbar, wie, verkürzt, Hampes Ruf nach mehr Vernunft vorhersehbar ist. Dennoch, der Revanchismus gegen die Eliten kommt nicht von Ungefähr: Es gibt auch in Deutschland keine Chancengleichheit und es liegt nicht nur an mangelnder Bildung oder Selbstermächtigung. Wieviel Prozent des Professoriums sind Arbeiterkinder? Wie viele Frauen, wie viele Migrantinnen und Migranten? Inwieweit liegt das nicht an fehlender Bildung, Wissen oder Willen, sondern daran, dass weitere, bourdieusche Distinktionsmerkmale fehlen, wie Kleidung, Benehmen und das Sprechen eines bestimmten Codes, den man im Plattenbau ungleich schwerer verinnerlicht als in der Doppelhaushälfte? Wieviel hängt daran, dass man bestimmten Gruppen, qua sozialem Stereotyp, auch bei gleicher Bildung, ganz im Sinne von Miranda Frickers Epistemic Injustice, weniger Wissen und Kompetenz zuschreibt (Fricker 2007)?

Vielleicht ist das alles implizit in Hampes Ruf nach "Autonomie der Lebensgestaltung" und in "Vermeidung von Grausamkeiten" (14). Ermächtigung hieße dann aber ganz explizit echte Teilhabe in Form von sozialem Aufstieg. An diese Möglichkeit muss man nicht nur glauben, es muss auch tatsächlich möglich sein, sonst handelt es sich um die Verbreitung von Illusionen, gegen die Hampe zurecht anschreibt. Ich denke, wir müssen uns auch der Frage stellen, inwieweit die gegenwärtige Krise nicht nur von den Potenzialen der sozialen Medien und einer "Erosion der aufgeklärten Kultur" (9) ausgelöst ist, sondern auch davon, dass die vergangenen Aufklärungen ihr Emanzipationsversprechen nicht gehalten haben - zumindest bei weitem nicht für alle.

5 Michael Hampe meint, die "Konkurrenz auf dem Arbeits-, Bildungs- oder Heiratsmarkt" (29) sitze einem falschen Analogieschluss vom Warenmarkt auf (30). Um Analogien bilden zu können, muss es eine Grenze zwischen den verschiedenen Bereichen, zwischen denen dann Analogien gebildet werden, geben. Ich frage mich, wie Hampe den Warenmarkt vom Rest unserer Wirklichkeit scheiden will. Wo Hampe unzulässige Analogieschlüsse sieht, sehe ich Kausalketten. Der "freie Markt macht die Autos besser und billiger und Brot und Fleisch allgemein zugänglich" (30), aber wie kann man die Produktionsbedingungen vom Rest unserer Lebenswelt trennen? Warum sind Fleisch und Brot so billig? Was zählt als Ware? Fleisch und Brot ja, aber die Liebe nicht? Wie stehts mit Arbeit? Die marxistische Tradition meint, das "mythische Denken," das Hampe denen unterstellt, die das "Marktdenken" universalisieren (30), liege genau in der Illusion, dass man Brot, Arbeit und Liebe trennen könne. ${ }^{2}$ Ich denke, eine dritte Aufklärung sollte schon mutiger sein, was das Kapital betrifft. ${ }^{3}$

Doch nun genauer zu Hampes Thesen unter den oben genannten zwei miteinander verbundenen Perspektiven: 1. Hampes Version des alten liberalen Paradoxons. 2. Hampes Verbindung von Grausamkeit mit Intensität. 


\section{Wehrhafte Aufklärung: wieviel Toleranz und Grausamkeit ist möglich und nötig?}

7 Michael Hampe widmet etwa ein Drittel seines Buches dem Thema Grausamkeit. Die "Vermeidung von Grausamkeiten" ist neben "Allgemeine[r] Bildung, Autonomie der Lebensgestaltung" und "Transparenz von Wissensansprüchen" Ziel "aufgeklärten Lebens" (14). Die Vermeidung von Grausamkeiten spielt moralisch eine fundierende Rolle: Geschichtlich waren die Bewegungen der Aufklärung "vor allem der Versuch, Grausamkeit einzudämmen, ja sie vielleicht irgendwann ganz zum Verschwinden zu bringen" (39). "Die Bekämpfung von Unterdrückung, Illusionen und Ungerechtigkeiten ist die Fortsetzung der Bemühung, Grausamkeiten zu vermeiden." (40). Hampe meint, und ich denke, das ist unkontrovers (aber eben nicht alles): "Es braucht Bildungsprozesse und den Mut, Evidenzen und Gründe einzufordern, um der Unterdrückung durch Illusionen und Unwahrheiten etwas entgegensetzen zu können." (40). Die Aufklärung darf dabei selbst nicht zur neuen Illusion werden (12) und das beinhaltet Klarheit darüber, was Hampe den "gemischten Charakter der Wirklichkeit" nennt: Historische Prozesse werden sowohl "von den Absichten der Menschen" als auch "von Gesetzmäßigkeiten" gesteuert (77), ohne dass wir unsere Absichten immer unter Kontrolle hätten (79).

Hier ist die Verbindung zu Hampes Variante des klassischen, liberalen Paradoxons: "Aufgeklärte Menschen versuchen, Grausamkeit als etwas zu vermeiden, das sich durch keine höhere Idee, ob sie nun aus der Religion oder dem Recht oder irgendeiner Ideologie stammt, rechtfertigen lässt." (50f.). Das beinhaltet natürlich die Aufklärung selbst - Gewalt und Grausamkeiten können nicht im Namen der Aufklärung verübt werden, aber um sich auf Argumente statt Gewalt einzulassen, muss man bereits aufgeklärt sein und den Gewaltverzicht unterschrieben haben. Wie aber wird man aufgeklärt bzw. gibt es ein Missionsziel und wie kann es durchgesetzt werden? Hampe scheint für eine Art Notwehr-Variante zu plädieren: "Gegen eine Argumenten unzugängliche Person, die die gewalttätige Auseinandersetzung sucht, kann man sich auch als Freund des Arguments nur mit Gewalt wehren. Erst wenn sie unterworfen ist, kann man davon absehen, ihr Grausamkeiten anzutun, sie zu versklaven oder zu töten, und ihr anbieten, sich doch auf das Argument einzulassen." (45). Das heißt, die Anhänger der Aufklärung "müssen bereit sein, eine Ausnahme von ihrer Lebensform zuzulassen" (45). Solange wir nicht angegriffen werden, gilt das Toleranzgebot (43).

Mit Rorty stellen sich folgende Fragen: Was genau sind Grausamkeiten? Sprechen wir hier nur von physischer Gewalt ("töten," "versklaven," "Krieg”)? Und warum Toleranz? Hampe verweist auch auf die "seelischen zwischenmenschlichen Grausamkeiten [...] wie sie etwa in ehelichen Beziehungen auftreten" (50), geht im Folgenden indes nicht näher darauf ein, sondern beschränkt seine Beispiele zuvörderst auf die Erkundung der Prädisposition $\mathrm{zu}$ und den möglichen Kontexten von physischer Gewalt und Grausamkeit.

Rortys Buch Contingency, Irony, and Solidarity (1989), auf das Hampe sich explizit bezieht, ist zur Hälfte Beschreibung der Figur der liberalen Ironikerin, die Rorty als ideale Einwohnerin seines liberalen Utopia sieht, und zur anderen Hälfte Beschreibung der Grausamkeit (cruelty), die dieser selbe Ansatz birgt. Rortys Buch ist ein Buch über Demütigung (humiliation), namentlich, die spezielle Art von Demütigung, die darin 
besteht, den existentiellen Referenzrahmen von Menschen zu zerstören - ihre Sinnmatrix.

11 In Rortys Lesart von Orwells 1984 zeigt sich O'Briens Sadismus nicht in erster Linie in den physischen Grausamkeiten, die er Winston antut, sondern darin, dass er in Winston jede Art der Sinngebung eliminiert, seine Liebe zu Julia zu bricht, ihm seine Sprache und damit jede Art der Verbindung zu anderen nimmt. Die besondere Grausamkeit O'Briens und des gesamten Systems Big Brother besteht für Rorty darin, die Menschen radikal zu vereinzeln, ihnen ihre Sprache zu nehmen, und sie damit letztlich ihres Selbsts zu berauben, das ein Selbst nur in Verbindung mit anderen sein kann - daher der Fall ins Bodenlose (Rorty 1989: 177f.).

Eine ähnliche, radikale Art der Vereinzelung stellt Gayatri Spivak in ihrem Aufsatz "The Rani of Sirmur" dar: Spivak beschreibt den Fall einer indischen Frau zur Kolonialzeit, die zusammen mit ihrem verstorbenen Ehemann verbrannt werden soll. Die Kolonialherren sind geschockt und wollen das im Namen der Aufklärung verhindern. Spivak beschreibt das Dilemma aus Sicht der Frau, um deren Schicksal es geht. Sie steht vor einer unmöglichen Wahl: Tod durch Verbrennen oder sozialer Tod durch Ausscheiden aus der eigenen Gruppe und sich unterwerfen unter den "Aufklärungsrahmen," der in ihrem Fall aber kein Leben, sondern nur bares Überleben garantieren würde - ein unmenschliches Leben, weil sie in keinen ihr verständlichen und existenziell lebbaren, sozialen Sinnkontext integriert wäre (Spivak 1985). Das Dilemma besteht darin, dass keiner der beiden Wege der Frau eine Existenz eröffnet und dass es keinen dritten Weg zu geben scheint.

13 Hampes Anspruch ist dezidiert "global" (Hampe 2018: 17, 22). Da stellen sich solche Fragen: Wen sprechen wir an und mit welchem Anspruch? Kommunikationsfähig zu machen (19), heißt nicht nur, Andere auf Argumentieren als Konfliktlösungsstrategie einzuschwören. Das alles weiß Hampe und spricht von emotionaler Bildung (23) und gemeinsamen Projekten als "säkulärer Sinnquelle" (32). Er führt allerdings nicht aus, wie diese existentielle Dimension, zumal auf globaler Ebene, genau aussehen könnte und wie der normative Rahmen konstituiert oder verändert wird - durch Aushandlung auf argumentativer Ebene? Implizit, durch das alltägliche Leben bestimmter, gemischter Normen?

Jenseits der individuellen Ebene stellt sich das Problem auch für Gruppen und auf intentionaler Ebene: Rorty meint, die meisten Menschen wollen sich und ihr Weltbild nicht ändern. Das ist das Problem, das Rorty mit seinem eigenen Konzept der "redescription" sieht: "redescription" benennt die Möglichkeit, sich stets in neue konzeptuelle Kleider zu kleiden, neue Referenzrahmen (vocabularies) zu schaffen. "But most people do not want to be redescribed. They want to be taken on their own terms taken seriously just as they are and just as they talk." (Rorty 1989: 89). Es ist eine Frage des Respekts vor der Würde der Anderen, sie sich selbst beschreiben zu lassen. "Redescription often humiliates." (Rorty 1989: 90).

15 Nun sollte man nicht vorschnell Rortys "liberale Ironikerin" mit Hampes "liberaler, aufgeklärter Kritikerin" gleichsetzen. Aber für beide, Hampe und Rorty, ergibt sich derselbe, oben genannte Deadlock: Jenseits der Definition von Grausamkeit, die Rorty um existentielle Demütigungen ergänzt, stellt sich das Problem der Kohabitation des Differenten. Wieviel Differenz geht vor dem eigenen Rahmen? Hampe plädiert, solange Nichtangriffspakte eingehalten werden, für Toleranz. Rorty plädiert für den "melting pot” (Rorty 1997: 100). Respekt darf für Rorty nicht zum Selbstläufer werden. Wenn 
man es auf Schlagworte niederbrechen möchte, könnte man sagen: Solidarity first! Difference second.

Rortys "liberal ironist" meint, dass

human solidarity is not a matter of sharing a common truth or a common goal but of sharing a common selfish hope, the hope that one's world - the little things around which one has woven one's final vocabulary - will not be destroyed. For public purposes it does not matter if everybody's final vocabulary is different, as long as there is enough overlap so that everybody has some words with which to express the desirability of entering into other people's fantasies as well as into one's own. (Rorty 1989: 92f.)

Rortys emotionale Bildung dient nicht Emanzipationszwecken (wie bei Hampe bspw. der Kinder gegenüber ihren Eltern, 23), sondern der sozialen Kohäsion. Rorty glaubt, wir brauchen "skill at imaginative identification" (93), die wir trainieren "by enlarging our acquaintances" (80). Dieser hermeneutische Prozess wirkt in beide Richtungen (Horizontverschmelzung), wenn auch startend von unserem westlichen Standpunkt (192). Was gestärkt werden soll, sind die "we-intentions" - dass wir uns als gemeinsame Gruppe verstehen - und dass wir uns, unter diesem gemeinsamen Schirm, der Fragilität unserer differenten Rahmen bewusst sind.

18 Auch Hampe reißt die Notwendigkeit des Gemeinschaftsgefühls an, wenn er davon spricht, dass wir gemeinsame Projekte brauchen, aber er führt nicht aus, was das im Einzelnen bedeutet, zumal, wie gesagt, auf globaler Ebene. Ich meine, hier einen Rest von utopischem Universalismus (die Aufklärung selbst [Rationalität?] wird für das Gemeinschaftsgefühl sorgen?) zu hören, den Rorty durch radikalen Partikularismus und Lokalismus ersetzt:

Pragmatists suggest that we simply give up the philosophical search for commonality. They think that moral progress might be accelerated if we focused instead on our ability to make the particular little things that divide us seem unimportant - not by comparing them with the one big thing that unites us but by comparing them with other little things. Pragmatists think of moral progress as more like sewing together a very large, elaborate, polychrome quilt, than like getting a clearer vision of something true and deep. [...] [T] hey hope to minimize one difference at a time - the difference between Christians and Muslims in a particular village in Bosnia, the difference between blacks and whites in a particular town in Alabama, the difference between gays and straights in a particular Catholic congregation in Quebec. The hope is to sew such groups together with a thousand little stitches - to invoke a thousand little commonalities between their members, rather than specify one great big one, their common humanity. (Rorty 1999: 86f.)

Hampe spricht, wie Rorty, von der Notwendigkeit "dem Leben kollektiv einen Sinn und eine kulturelle Gestalt zu geben" (21), die sich nicht im Verweis auf unsere gemeinsame Biologie oder eine gemeinsame, statische Wahrheit erschöpfen könne. Für Rorty ist wichtig, dass wir, jeweils in unseren kleinen Gemeinschaften, immer wieder in den gleichen oder zumindest kompatible Imaginationsräume eintreten.

Die Menschen am kollektiven Imaginationsraum "Aufklärung" teilhaben zu lassen, ist für Rorty zudem intrinsisch verbunden mit echter Chancengleichheit: "To retain social hope [...] [people] need to be able to tell themselves a story about how things might get better, and to see no insuperable obstacles to this story's coming true." (Rorty 1989: 86). Momentan gilt das in Europa für den weitaus kleineren Teil der Bevölkerung. Hampes Wahrheitspraktiken sind wichtig, aber damit die dritte Aufklärung über die 
zweite hinausgeht, müsste sie für Rorty, neben der von Rorty geforderten Einfühlung und Empathie, auch ganz konkret die Umverteilung von Ressourcen beinhalten. Zunächst in der eigenen Gruppe, beispielweise innerhalb Deutschlands und zwischen Deutschland und Europa, wenn wir, wie ich, Europa als unsere Gruppe sehen. Die Geschichten, die wir erzählen, müssen stimmen und damit die Geschichten der besseren Zukunft in Europa keine Illusionen bleiben, sondern wahr werden (können), müssen wir Solidarität wagen! Materiell! Es wäre interessant, welche Ideen Hampe, wenn er übereinstimmt, dass "Autonomie der Lebensgestaltung" und "Vermeidung von Grausamkeiten" (14) Chancengleichheit beinhaltet und materiell gesichert sein muss, hier global hat und ob diese nicht seinem Bekenntnis zum Marktliberalismus widersprechen.

\section{Grausamkeit und intensive Erfahrung}

21 Michel Hampe beleuchtet das Konzept der Grausamkeit nicht nur von Seiten der normativen Rechtfertigung einer politischen Ordnung - es geht nicht nur darum, wann wir Gewalt und Grausamkeit, als Ausnahme, zulassen dürfen und müssen. Hampe versucht das Konzept Grausamkeit an sich zu beleuchten, um, wenn die Quellen verstanden sind, Wege zu haben, Grausamkeit zu verhindern.

Hampe schreibt, es haben sich, mit Ausnahme Judith Shklars und Richard Rortys, "nur wenige Wissenschaftler" (50) dem Thema "Grausamkeit" gewidmet, wobei er mit Wissenschaft wahrscheinlich die "rationale [...] Philosophie" meint, zu der er selbst beiträgt (12). ${ }^{4}$ Vielleicht liegt der Grund dafür darin, dass Grausamkeit nicht (nur) auf rationaler Ebene arbeitet - es geht nicht um Gründe, Illusionen und ihre Aufhebung -, sondern grundlegend die affektive Dimension betrifft. Hampe beschreibt Grausamkeit als "Laster" - als etwas also, das man sich, trotz Verbots, erlaubt. Das passt in den Kantischen Rahmen der Affektbeherrschung. Grausamkeit ist der Ausbruch aus diesem Disziplinierungsraum.

Rorty nimmt, Annette Baier folgend, keine strikte, sondern nur eine graduelle Grenze "between reason and sentiment, thinking and feeling" (Rorty 1999: 75) an. Für Rorty ist das Selbst ein "centerless web of beliefs and desires" (Rorty 1989: 88). Eine weitere Aufklärung beinhaltet für Rorty auch, das kantische Erbe dahingehend zu überwinden, dass wir unsere Gefühle nicht als streng geschieden von unseren Gedanken sehen. ${ }^{5}$ Unser "final vocabulary" ist konstituiert durch unsere "beliefs and desires" ebenso wie unsere Hoffnungen, unser Wissen, was wir für möglich und nötig erachten, unsere Beziehungen zu anderen. "From this point of view, moral progress is not an increase of rationality - a gradual diminution of the influence of prejudice and superstition, permitting us to see our moral duty more clearly. [...] [I]t is best to think of moral progress as a matter of increasing sensitivity, increasing responsiveness to the needs of a larger and larger variety of people and things." (Rorty 1999: 81).

Baier argumentiert dafür, den Begriff der "moral obligation" durch den des “appropriate trust" zu ersetzen (Baier 1985: 232, zitiert in Rorty 1999: 76). Es sei, "entirely natural" (auch wenn hier Ausnahmen und Abweichungen die Regel bestätigen mögen) sich um seine Familienangehörigen zu kümmern (Rorty 1999: 78), wobei "coming natural" (inclination) das Gegenteil von "obligation" ist. Rorty sieht unsere Aufgabe darin, diesen Kreis zu erweitern: 
Moral development in the individual, and moral progress in the human species as a whole, is a matter of re-making human selves so as to enlarge the variety of the relationships which constitute those selves. [...] The term "moral obligation" becomes increasingly less appropriate to the degree to which we identify with those whom we help: the degree to which we mention them when telling ourselves stories about who we are, the degree to which their story is also our story. (Rorty 1999: 79)

Hampe adressiert die beiden Seiten des Körper-Geist-Dualismus, wenn er von der Notwendigkeit von Argumenten und Erfahrungen schreibt: "Das Ziel aufklärerischer Bewegungen war stets: Reden, mit Argumenten streiten, statt die Armeen zu mobilisieren; Menschen $\mathrm{zu}$ bilden, damit sie andere Quellen intensiver Erfahrung erschließen können als die Grausamkeit." (Hampe 2018: 64). Intensität scheine, laut Hampe, verbunden $\mathrm{zu}$ sein mit der Verletzlichkeit des menschlichen Lebens Bergsteigen, auch Kriege erklären sich so (63).

[...] [F]ür das Streben nach Intensität steht auch die ästhetische Erfahrung. Diejenigen Personen, die anders als Montaigne dem Spektakel der Grausamkeit [bspw. öffentlichen Hinrichtungen] mit Freude beiwohnen oder es gar mit Freude selbst veranstalten, suchen eine spezielle Intensität ihrer Erfahrung beim Ausführen oder Anblick grausamer Handlungen, weil sie kaum über andere Möglichkeiten der intensiven Wirklichkeitserfahrung verfügen. Für Montaigne mag das anders gewesen sein, weil ihm andere ästhetische Erfahrungen von hoher Intensität möglich waren, etwa in der Wahrnehmung von Musik, Malerei oder Literatur. (63f.)

Rorty meint, Nabokov zeige, es gebe eine "special sort of cruelty of which those capable of bliss are also capable. [...] [Nabokov's] books are reflections on the possibility that there can be sensitive killers, cruel aesthetes, pitiless poets" (Rorty 1989: 157); "there is no synthesis of ecstasy and kindness" (160).

Das Bildungsbürgertum ist für Rorty nicht weniger anfällig für Grausamkeit als andere Bevölkerungsgruppen. Grausamkeit kann gar selbst zur Kunstform werden. In Rortys Lesart von Orwells 1984 erlaubt O'Briens Intelligenz und Bildung gerade die Virtuosität seiner speziellen Art von Folter, die ich unter 1. beschriebenen habe: die Zerstörung von existentiellen Sinnmatrizen, die Wissen und auch eine bestimmte Art der Einfühlung ${ }^{6}$ voraussetzt. “The only object of O'Brien's intensive seven-year-long study of Winston was to make possible the rich, complicated, delicate, absorbing spectacle of mental pain which Winston would eventually provide." (179). Rorty meint: "The point of breaking Winston is not to bring Winston into line with the Party's ideas. The Inner Party [...] is torturing Winston for the sake of causing Winton pain, and thereby increasing the pleasure of its members, particularly O'Brien." (179). Das Zufügung von Leid ist Endzweck, ganz ähnlich wie Hampe Achills Blutrausch als Selbstzweck versteht. Hampe meint: "Die Kriegssituation bietet die Möglichkeit, Freude an der Leidverursachung und dem Töten anderer $\mathrm{zu}$ entwickeln und dies vor sich $\mathrm{zu}$ verschleiern, indem man es als notwendige Kampfhandlung, die vermeintlich einem Kriegsziel dient, ausgibt." (60).

Orwells posttotalitärer Staat ist, in Rortys Lesart, frei von diesen sekundären Rechtfertigungsstrategien:

Torture is not for the sake of getting people to obey, nor for the sake of getting them to believe falsehoods. As O'Brien says, "The object of torture is torture." For a gifted and sensitive intellectual living in a posttotalitarian culture, this sentence is the analogue of "Art for art's sake" or "Truth for its own sake," for torture is now the only art form and the only intellectual discipline available to such a person. (Rorty 1989: 180) 
Rorty meint, Folter sei das zentrale Thema von 1984 und Wahrheit nur "a red herring" (182). Die ganze Realismusdebatte lenke von Orwells viel größerem Verdienst ab, das darin bestehe, O’Brien als mögliche Figur dargestellt zu haben (176). Im Rahmen dieses Symposiums verstehe ich das so: Wir sollten nicht nur gegen die Erosion der aufgeklärten Kultur arbeiten, auch wenn ich das unterschreibe, sondern auch gegen die Erosion der demokratischen Institutionen.

\section{Schluss: Aufklärung, Demokratie und Intervention}

Demokratie ist für mich nicht, wie für Michael Hampe in vorliegendem Text, nur die Herrschaft der Mehrheit, sondern inkludiert den Schutz der Minderheit, Pluralismus, Gewaltenteilung und einiges weiteres. Ich finde Hampes konzeptionelle Trennung von aufgeklärter Kultur und Demokratie und die höhere Gewichtung der aufgeklärten Kultur über den demokratischen Staatsapparat theoretisch interessant, aber aus den vorherigen Argumenten ergibt sich eine stärkere Verbindung zwischen beiden.

Zuspitzungen dieser Art mögen der Textform Essay - die tastend auch kontroverse Gedanken ausloten darf - geschuldet sein. Handelt es sich aber um einen Essay? Wer sind die Adressatinnen und Adressaten? Was ist Hampes Ziel mit diesem Text?

Hampe schreibt an einer Stelle, er verorte sein Buch im Kontext der pragmatischen Philosophie, die "ihre Äußerungen [...] nie nur als Beschreibungen der Tatsachen, sondern auch als mögliche Interventionen [versteht], die den Lauf der Dinge mitbestimmen und verändern könnten, wenn sie eine entsprechende Wirkung entfalten" (Hampe 2018: 69). Ich habe den Eindruck, dass sich Hampes Buch, qua Verlagswahl und Rhetorik vor allem an die eigene Gruppe, die aufgeklärte Lebensweisen ohnehin gutheißt und daher keine Intervention benötigt, richtet. Um breitere Wirkung zu entfalten, müsste, ganz Rortyanisch, der Aufklärungsrahmen mit den Sehnsuchtsräumen derer, die wir an Bord haben wollen, verknüpft werden und diese Sehnsuchtsräume dürfen nicht in Utopia verortet sein, sondern müssen in der Wirklichkeit liegen.

\section{BIBLIOGRAPHY}

ARRUZZA Cinzia, BHATTACHARYA Tithi \& Nancy FRASER, (2019), Feminism for the 99\%, A Manifesto, London, Verso.

BAIER Annette, (1985), Postures of the Mind, Minneapolis, University of Minnesota Press.

FRICKER Miranda, (2007), Epistemic Injustice, Power and the Ethics of Knowing, Oxford, Oxford University Press.

HAMPE Michael, (2018), Die Dritte Aufklärung, Berlin, Nicolai Publishing and Intelligence GmbH.

ILLOUZ Eva, (2011), Warum Liebe weh tut, Eine soziologische Erklärung, Berlin, Suhrkamp. 
MARX Karl, (1962), Das Kapital, Kritik der politischen Ökonomie, Erster Band, Buch I, Der

Produktionsprozeß des Kapitals, Berlin, Karl Dietz Verlag.

RORTY Richard, (1989), Contingency, Irony, and Solidarity, Cambridge, Cambridge University Press.

RORTY Richard, (1997), Achieving Our Country, Cambridge, Ma., Harvard University Press.

RORTY Richard, (1998), "Human Rights, Rationality, and Sentimentality," in Id., Truth and Progress, Cambridge, Cambridge University Press, 167-85.

RORTY Richard, (1999), "Ethics without Principles," in Id., Philosophy and Social Hope, London, Penguin Books, 72-90.

SPIVAK Gayatri Chakravorty, (1985), "The Rani of Sirmur, An Essay in Reading the Archives,"

History and Theory, 24 (3), 247-72.

\section{NOTES}

1. Bzw. wenn Hampe die Affekte anspricht, scheint es sich um ihre funktionale Rolle im Streben nach Autonomie (etwa der Kinder gegenüber ihren Eltern, Hampe 2018: 23) oder um ihre (rationale) Verfeinerung weg von den kruden Trieben zu handeln (bspw. wenn Montaignes ästhetisches Erleben gepriesen wird, 63). Ich werde auf beides zurückkommen.

2. Vgl. bspw. Marx 1962, Illouz 2011.

3. Auch müssten weitere Themen, bspw. Ökologie und Feminismus, einbezogen werden. Das Problem ist, dass es dann oft schnell beliebig wird. Ich bin allerdings, mit Arruzza, Bhattacharya und Fraser (2019) überzeugt, dass die Themen analytisch nicht trennbar sind. Was politisch die richtige Strategie der Durchsetzung ist, steht wieder auf einem anderen Blatt.

4. Ich kenne mich nicht aus, kann mir aber nicht vorstellen, dass das Phänomen "Grausamkeit" in anderen Disziplinen nicht durchaus gut beforscht wäre, bspw. in der Psychologie oder Soziologie.

5. Rorty plädiert, Hampes erste, sokratische Aufklärung nicht mitzählend, Putnam folgend, immer wieder für eine "Zweite" Aufklärung.

6. Das spräche auch gegen Rortys Einfühlungs-basierten Ansatz: zu fühlen, dass der andere leidet, scheint Voraussetzung des Lustgewinns des Folterers zu sein. Rorty würde wahrscheinlich sagen, wir wenden diese Art von Grausamkeit nur gegen Menschen an, die wir nicht als unsereins sehen, mit denen wir nicht über eine gemeinsame Narration verbunden sind (siehe dazu: Rorty 1998).

\section{AUTHOR}

\section{YVONNE HÜTTER-ALMERIGI}

Università di Bologna

yvonne.huetter[at]gmx.net 